\title{
Erratum: Noiseless intensity amplification of repetitive signals by coherent addition using the temporal Talbot effect
}

Reza Maram, James Van Howe, Ming Li \& José Azaña

Nature Communications 5:5163 doi: 10.1038/ncomms6163 (2014); Published 16 Oct 2014; Updated 19 Nov 2014

This Article contains an error in Fig. 1 that was introduced during the production process. The pulse waveforms were displayed in white colour, making some of them invisible against the background. The correct version of Fig. 1 appears below.

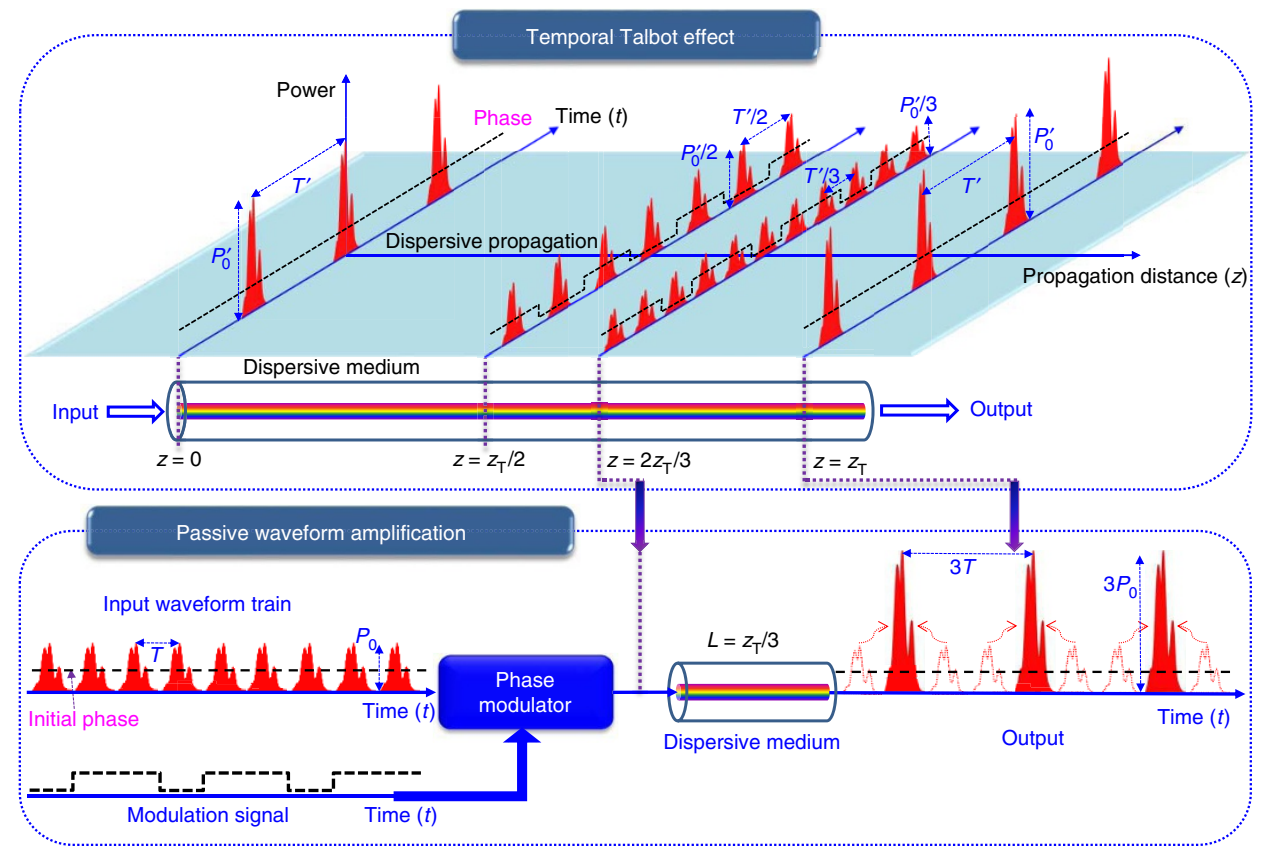

Figure 1 